\title{
Towards Improved Reliability of the Analysis of Factors Influencing the Properties on Steel in Industrial Practice
}

\author{
Tatjana VECKO-PIRTOVSEK, ${ }^{1)}$ Iztok PERUS, ${ }^{2)}$ Goran KUGLER ${ }^{1)}$ and Milan TERCELJ ${ }^{1)}$ \\ 1) Faculty of Natural Sciences and Engineering, Department of Materials and Metallurgy, University of Ljubljana, Aškerčeva \\ cesta 12, 1000 Ljubljana, Slovenia. E-mail: milan.tercelj@ntf.uni-lj.si \\ 2) Faculty of Civil and Geodetic Engineering, University of Ljubljana, Jamova 2, 1000 Ljubljana, Slovenia.
}

(Received on July 17, 2008; accepted on December 27, 2008)

\begin{abstract}
Due to the large number of parameters that influence the properties of steel and lower accuracy of some measured data, it can take several years for industry to collect a database large enough to carry out reliable analysis. In this paper a new approach is presented to overcome these problems. The Latin hypercube sampling technique (LHS) was used for modelling of the uncertainty of measured industrial data and CAE NN was used for analysis of the mutual dependence of influencing parameters. Using the example of AISI D2 tool steel, the proposed method was applied for determination of relationships between chemical composition and yield $\delta$ by considering the corresponding coefficients of variation. New insights of relationships were directly applicable in the industrial practice. Despite using small database, it was possible to considerably increase the yield $\delta$ for industrial hot rolling.
\end{abstract}

KEY WORDS: metal production; D2 tool steel; Latin hypercube sampling; measurement uncertainty; neural network; hot deformability.

\section{Introduction}

In the production of tool steels the problem of lower hot deformability, one of the most essential steel properties, should first be solved since it influences the mechanical properties, yield $\delta^{*}$ and consequently the economics of the production process. Hot deformation of steels can be studied experimentally by compression, tensile and torsion testing. It can also be studied by numerical analysis and by modelling of particular aspects of hot deformation. In this way the approximate values about major technological process parameters resulting in acceptable mechanical properties can be obtained. Such parameters, for example, are the upper and lower limit, the optimal temperature range, ${ }^{1-3)}$ appropriate reduction of height, and the plasticity limit, ${ }^{4-8)}$ strains, stresses, and temperature field. ${ }^{9)}$ On the other hand, modelling can be used to obtain general relationship between microstructure and mechanical properties. ${ }^{10)}$ Thus in this way the production process may be well established. For further considerable improvement of the hot deformation process the variable nature of steel production should be considered too, i.e. additional knowledge about relationships between the most influential parameters and deformability (yield $\delta$ ) is needed. Further, it is very important that reliable analysis of the influences of processing parameters and chemical composition on hot deformability of steels can already be carried out in the early stage of production, i.e. with a small database containing data with lower accuracy. In the works of Dobrzanski, ${ }^{11)}$ Çöl et al., ${ }^{12)}$ Capdevila et al., ${ }^{13)}$ it was pointed out that due to the large number of parameters influencing steel properties several years of database collecting is usually needed in order to be able to carry out reliable analysis. Furthermore, several authors such as Sandberg, ${ }^{5)}$ Imai et al., ${ }^{14)}$ argued that some chemical elements (e.g. $\mathrm{Ti}, \mathrm{Nb}, \mathrm{Pb}, \mathrm{Sn})$ could have a decisive influence on the final properties, as well as on hot deformability, despite their very low concentration.

Thus insufficient knowledge about the influence of relevant industrial process parameters and chemical composition of tool steels on steel properties on one hand and the uncertainty of measured parameters in industry on the other, prevent us from effectively achieving constancy of mechanical properties and hot deformability. When dealing with complex mutual dependence of many influencing parameters, neural networks are often used (see Fig. 1). In the literature few publications dealing with analysis of several parameters influencing metal properties based either on laboratory or industrial data (sheet rolling of low carbon steels, etc.) are known; especially the latter usually exhibit complex relationships between parameters and the final properties of steel (see e.g. Refs. 11)-13),15)-17)).

Analysing the industrial database represents a huge re-

* The yield $\delta$ in present study is the measure for occurrence of surface cracking during hot rolling process, and refers to the ratio between successfully processed material and successfully plus unsuccessfully processed material. 


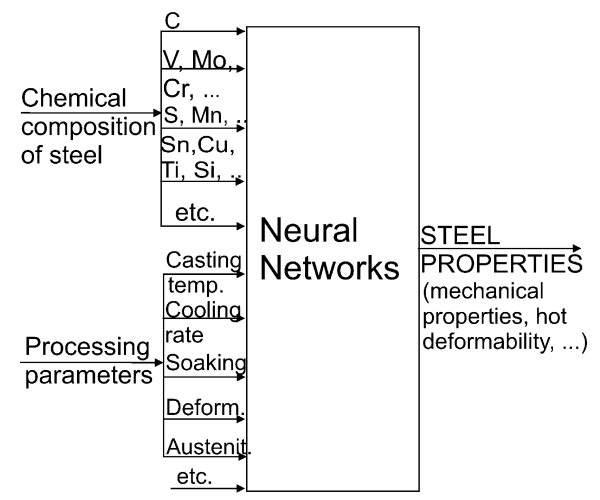

Fig. 1. Schematic diagram of neural network approach for complex analysis of influential parameters as well as for prediction of the properties of tool steels.

search challenge. Namely, using the industrial database that contains the essential parameters of steel production and also taking into consideration the uncertainty of the measured data requires appropriate methods of data analysis. In this way reliable optimization can be carried out already in the early stage of production. This paper deals with analysis of the influence of chemical composition on the yield $\delta$ of D2 tool steel using a small industrial data base. The approach takes into account the uncertainty of the measured data employing the Latin hypercube sampling technique, especially at the lower and upper range of their allowable variation. According to the statement of Mandal et al., ${ }^{18)}$ that neural networks should contribute to the improvement of their explanatory insights and not only be used as a "black box" approach, the present work is intended to gain new insight into the relationships between chemical composition and yield $\delta$, and the resulting in increase in the yield $\delta$ of industrial hot rolling.

\section{Experimental: Database on Hot Deformability}

In order to carry out the analysis an industrial database containing chemical composition and yield $\delta$, which was taken as a measure of hot deformability of rolled AISI D2 tool steel, was constructed. The database contains 72 hotrolled samples. The random variation of chemical composition in the allowable range covered the entire problem space, though the majority of the information was concentrated somewhere in the middle of the allowable intervals of variation for a single element.

Ingots with initial dimensions $400 \mathrm{~mm} \times 400 \mathrm{~mm}$ were hot rolled in 20 passes into squares of $200 \mathrm{~mm} \times 200 \mathrm{~mm}$. The soaking temperature was $1200^{\circ} \mathrm{C}$ and pieces were rolled in the temperature range $1180-900^{\circ} \mathrm{C}$. The yield $\delta$ percentage during hot rolling varied in the interval of 78-95\%. The concentrations of chemical elements ( $\mathrm{Si}, \mathrm{Cr}$, $\mathrm{V}, \mathrm{Mn}, \mathrm{Mo}, \mathrm{W}$, etc.) were determined by optical emission spectroscopy (OES) with accuracy between $0.01 \%$ and $0.1 \%$. The carbon content was determined by the ignition method in an induction furnace and an infrared absorber detector (LECO). The typical accuracy of the chemical analysis equipment for carbon given by producers is about $1 \%$. Table 1 gives mean values of chemical elements, methods of measurement, uncertainties $(2 \sigma)$ of measurements and the corresponding coefficients of variation
Table 1. Chemical elements contained in AISI D2 tool steel, with mean values, methods of measurement, uncertainty in measurement $(2 \sigma)$ and corresponding coefficients of variation $(\mathrm{CoV})$.

\begin{tabular}{|c|c|c|c|c|}
\hline Element & $\begin{array}{c}\text { Mean } \\
\text { values } \\
{[\%]}\end{array}$ & Method & $\begin{array}{c}\text { Uncertainty } \\
\mathbf{( 2 \sigma )}\end{array}$ & $\begin{array}{c}\mathbf{C o V} \\
{[\%]}\end{array}$ \\
\hline $\mathrm{C}$ & 1.534 & LECO & 0.020 & 0.7 \\
\hline $\mathrm{Si}$ & 0.277 & OES & 0.020 & 3.6 \\
\hline $\mathrm{Mn}$ & 0.230 & OES & 0.020 & 4.3 \\
\hline $\mathrm{P}$ & 0.025 & OES & 0.002 & 4.1 \\
\hline $\mathrm{S}$ & 0.008 & LECO & 0.001 & 6.5 \\
\hline $\mathrm{Cr}$ & 11.359 & OES & 0.100 & 0.4 \\
\hline $\mathrm{Ni}$ & 0.205 & OES & 0.010 & 2.4 \\
\hline $\mathrm{Mo}$ & 0.743 & OES & 0.020 & 1.3 \\
\hline $\mathrm{V}$ & 0.836 & OES & 0.030 & 1.8 \\
\hline $\mathrm{Al}$ & 0.015 & OES & 0.004 & 13.8 \\
\hline $\mathrm{Cu}$ & 0.181 & OES & 0.010 & 2.8 \\
\hline $\mathrm{As}$ & 0.016 & OES & 0.003 & 9.6 \\
\hline $\mathrm{Co}$ & 0.011 & OES & 0.010 & 44.1 \\
\hline $\mathrm{W}$ & 0.013 & OES & 0.020 & 79.1 \\
\hline $\mathrm{Sn}$ & 0.039 & OES & 0.002 & 2.5 \\
\hline $\mathrm{Ti}$ & 0.008 & OES & 0.020 & 123.9 \\
\hline Notes: LECO: ignition method in an induction furnace;
\end{tabular}

$(\mathrm{CoV})$; this is defined as the ratio between the standard deviation $\sigma$ and the mean value. $2 \sigma$ for the yield $\delta$ was set as $1 \%$.

\section{Modelling of Hot Deformability}

\subsection{Basics of a CAE Neural Network}

A prerequisite condition for spatial analysis is the choice of appropriate method. In our case we used CAE NN, which makes possible relatively simple modelling of the mutual interactions of particular chemical elements.

The hot deformability (i.e., expressed in terms of the yield, $\delta$ ) of a tool steel is determined by observing $N_{\mathrm{mv}}$ specimens of tool-steel ingots. The mathematical description of the observation of a single specimen is called a model vector. As a result, the entire phenomenon can be described by a finite set of $N_{\mathrm{mv}}$ model vectors

$$
\left\{\mathbf{X}_{1}, \ldots, \mathbf{X}_{n}, \ldots, \mathbf{X}_{N_{\mathrm{mv}}}\right\}
$$

By using the conditional probability density function, ${ }^{19)}$ the optimal estimator which estimates some unknown properties of the phenomenon of hot deformability on the basis of an incomplete observation obtained with a previous complete observation can be expressed as

$$
\begin{aligned}
c_{k} & =\sum_{n=1}^{N_{\mathrm{mv}}} A_{n} \cdot c_{n k}, \quad \text { where } A_{n}=\frac{a_{n}}{\sum_{i=1}^{N_{\mathrm{mv}}} a_{i}} \text { and } \\
a_{n} & =\frac{1}{(2 \pi)^{D / 2} w^{D}} \exp \left[-\sum_{l=1}^{D} \frac{\left(b_{l}-b_{n l}\right)^{2}}{2 w^{2}}\right]
\end{aligned}
$$

where $c_{k}$ is the estimate of the $k$-th output variable (i.e. yield $\delta), c_{n k}$ is the same output variable corresponding to the $n$-th model vector in the database, $N_{\text {mv }}$ is the number of model vectors in the database, $b_{n l}$ is the $l$-th input variable of the $n$ th model vector in the database $\left(e . g ., b_{n 1}, b_{n 2}, b_{n 3}, \ldots, b_{n \mathrm{~L}}\right)$, and $b_{l}$ is the $l$-th input variable corresponding to the prediction vector. $D$ is the number of input variables, and defines the dimensions of the sample space. The Gaussian function 
is used for smooth interpolation between the points of the model vectors. In this context the width $w$ is called the "smoothing" parameter.

An intermediate result in the above described computational process is the estimated probability density function $\hat{\rho}$ of known input variables

$$
\rho=\frac{1}{N} \sum_{n=1}^{N} a_{n}
$$

It represents a measure of how the influence of all the specimens in the database is spread over the sample space and it strongly depends on the smoothing parameter $w$. It has important practical meaning since it helps to detect possibly less accurate predictions due to the data distribution in the database and due to local extrapolation outside the data range. The higher the $\hat{\rho}$ value, the more specimens (relative to the total number of specimens in database) with input variable similar to the input variable of the prediction vector exist in the database. Consequently, the prediction is more reliable and vice versa.

\subsection{Consideration of the Uncertainty in the Industrial Data}

Uncertainty is a quantitative indication of the quality of a measured (industrial) result. It gives an answer to the question of how well the result represents the value of the quantity being measured. Fortunately, the database obtained includes data on the uncertainty due to measurement error with the assumed probability density distributions. This is additional important information which allows the study not only of the influence of chemical elements themselves, but also the influence of uncertainty in measurement. This is of particular interest when the content of a chemical element is low. Unfortunately, in such cases, $\mathrm{CoV}$ is high due to the error measurements.

Among different statistical techniques, the method of Latin hypercube sampling (LHS) ${ }^{20)}$ was used to generate a distribution of plausible collections of parameter values from a multidimensional distribution. The technique was first described by McKay et $a l^{20)}$ and then further elaborated by Iman et al. ${ }^{21)}$ In the context of statistical sampling, a square grid containing sample positions is a Latin square if and only if there is only one sample in each row and each column. A Latin hypercube is the generalisation of this concept to an arbitrary number of dimensions, whereby each sample is the only one in each axis-aligned hyperplane containing it.

When sampling a function of $N$ variables, the range of each variable is divided into $M$ equally probable intervals. $M$ sample points are then placed to satisfy the Latin hypercube requirements; note that this forces the number of divisions, $M$, to be equal for each variable. Also note that this sampling scheme does not require more samples for more dimensions (variables); this independence is one of the main advantages of this sampling scheme. Another advantage is that random samples can be taken one at a time, remembering which samples were taken so far. In Latin hypercube sampling one must first decide how many sample points to use and for each sample point remember from which row and column the sample point was taken. LHS ensures that the ensemble of random numbers is representative of the real variability.

In the present study the number of variables $N$ was equal to 17 (=16 chemical elements +yield $\delta$ ) and $M$ was equal to 50 (a relatively high number, which was chosen for the best description of the phenomenon). Normal probability density distributions were assumed for all variables (e.g. chemical elements) according to the given data from the measurements. An increased database was generated which consisted of 3600 model vectors instead of 72 in the original database. 50 model vectors $(M=50)$ were generated around each original model vector and the same procedure was repeated 72 times (for each model vector in the original database).

\subsection{Analysis of the Influences on Hot Deformability}

When using CAE for an estimate of hot deformability (yield $\delta$ ) $, \hat{\delta}, \delta_{n}$ is the only output parameter $(k=1)$ and, in the majority of cases presented in the paper, is a function of only two input parameters. For example, in the case of the influence of the percentage of sulphur and the percentage of manganese on the yield $\delta$ of hot deformability, the $n$-th steel ingot in the database is represented by a model vector, where the variables $b_{n 1}, b_{n 2}$ and $c_{n 1}$ represent $\% \mathrm{~S}_{n}, \% \mathrm{Mn}_{n}$, and $\delta_{n}$, respectively, while the steel ingot under consideration is represented by a prediction vector, where the variables $b_{1}, b_{2}$ and $\hat{c}_{1}$ represent $\% \mathrm{~S}, \% \mathrm{Mn}$ and $\hat{\delta}$, respectively. $a_{n}$ in Eq. (2) for this particular case can be rewritten simply as

$$
\begin{aligned}
a_{n}= & \frac{1}{2 \pi \cdot w_{n, \% \mathrm{~S}} \cdot w_{n, \% \mathrm{Mn}}} \\
& \times \exp \left[-\frac{\left(\% \mathrm{~S}-\% \mathrm{~S}_{n}\right)^{2}}{2 w_{n, \% \mathrm{~S}}^{2}}-\frac{\left(\% \mathrm{Mn}-\% \mathrm{Mn}_{n}\right)^{2}}{2 w_{n, \% \mathrm{Mn}}^{2}}\right] \ldots
\end{aligned}
$$

A non-constant smoothing parameter $w$, as suggested by Peruš et $a l .{ }^{22)}$ and Turk et al., ${ }^{23)}$ was used in the present study. Using a trial-and-error procedure where $w_{\min }$ and $w_{\max }$ were varied, $w_{\min }=0.07$ and $w_{\max }=0.15$ were selected as the most appropriate values for the smoothing parameter.

The CAE approach requires an appropriate database and a numerical analysis for each estimate. Note that the original database was increased by using the LHS (Latin hypercube sampling). This is certainly a drawback compared to closed-form equations derived from a traditional regression analysis. On the other hand, the advantage of the CAE approach is its flexibility. There are no fixed functional relations between the input and output parameters. Any number of input parameters (which are contained in the database) can be used, and different databases or different subsets of a database can be employed. Whereas closed-form empirical equations may be more convenient for practical applications, the CAE approach can be a very powerful tool in research.

\section{Results of the Analysis and Discussion}

At this point it should be stressed that the allowable variation of chemical composition of almost all chemical elements is very narrow, and as was already mentioned, the relationships between influential parameters and steel proper- 
ties are very complex, so consequently a clear relationship, especially at their lower and upper range of allowable variation, cannot be expressed with certainty. Some degree of continuity with the relationship of data in the middle of the variation range may also be found at the lower and upper range of variation. The results for yield $\delta$ are presented in terms of isolines representing constant values of estimated yield $\delta$ (e.g. Figs. 2-7, 9). As additional information, isolines of $\hat{\rho}$ values (Eq. $(3)^{22)}$ ), which are related to the reliability of the predictions, have been plotted (using thinner lines and smaller fonts). The larger the $\hat{\rho}$ value, the more reliable are the results, and vice versa. Note also, that in the predictions made by CAE, the same non-constant $w$ $\left(w_{\min }=0.07, w_{\max }=0.15\right)$ was used throughout the paper.

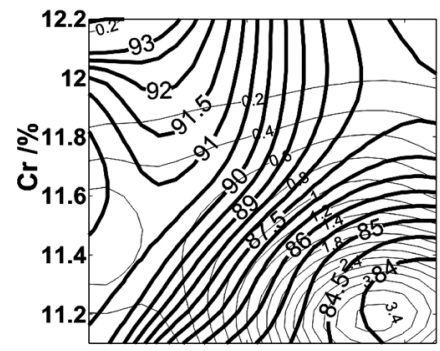

(a)

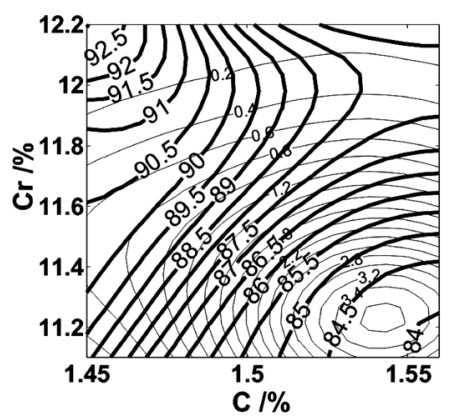

(b)

Fig. 2. Lines of equal yield $\delta$ s depending on $\mathrm{C}$ and $\mathrm{Cr}$ contents: (a) original database, (b) database increased by LHS. The $\hat{\rho}$ isolines are also shown (thin lines).

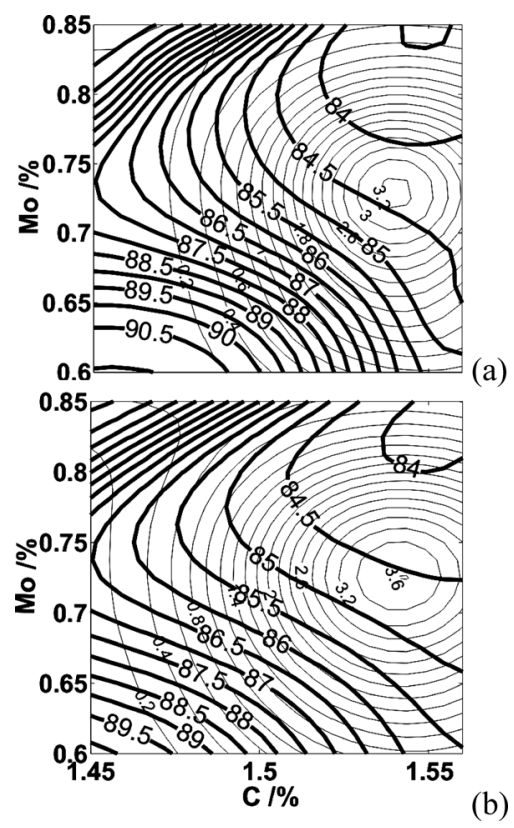

Fig. 3. Lines of equal yield $\delta$ depending on $\mathrm{C}$ and Mo contents: (a) original database, (b) database increased by LHS. The $\hat{\rho}$ isolines are also shown (thin lines).
Figures 2-9 show the complexity of the relationships between various pairs of chemical elements with various $\mathrm{CoV}$ and yield $\delta$. The $\mathrm{CoV}$ for each measured chemical element is given in Table 1 and amounts to $0.7 \%$ for $\mathrm{C}, 0.4 \%$ for $\mathrm{Cr}$, 1.3 for Mo, $4.3 \%$ for Mn, 6.5\% for S, $2.5 \%$ for Sn, $2.8 \%$ for $\mathrm{Cu}, 79.1 \%$ for $\mathrm{W}$ and $123.9 \%$ for $\mathrm{Ti}$, respectively. We can conclude that $\mathrm{CoV}$ for $\mathrm{Cr}$, Mo and $\mathrm{C}$ are very low, moderate for $\mathrm{Ni}, \mathrm{Mn}, \mathrm{S}, \mathrm{Cu}$ and $\mathrm{Sn}$, and high for $\mathrm{W}$ and $\mathrm{Ti}$. Note that a small value of $\mathrm{CoV}$ involves a small uncertainty, while a larger value of $\mathrm{CoV}$ involves a larger uncertainty.

From Fig. 2(a) it is apparent that an increased content of $\mathrm{C}$ at the same level of $\mathrm{Cr}$ decreases the yield $\delta$ of hot rolling, while increased $\mathrm{Cr}$ results in an increased yield $\delta$.

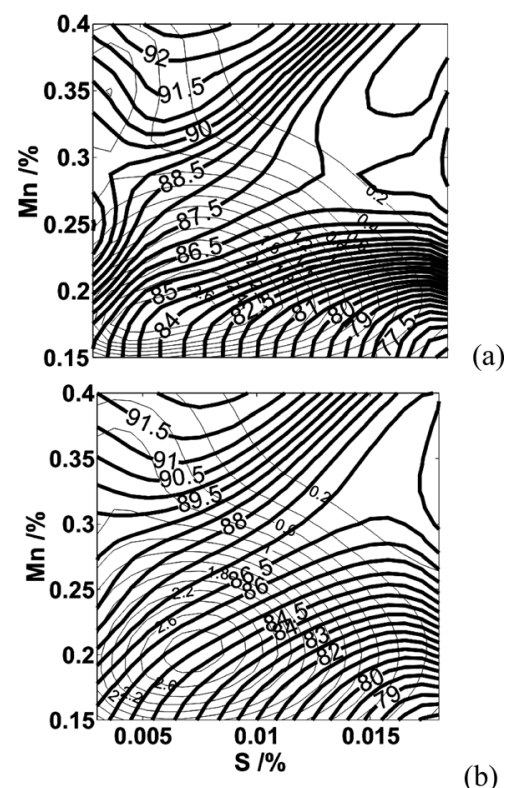

(b)

Fig. 4. Lines of equal yield $\delta$ depending on $\mathrm{S}$ and Mn contents: (a) original database, (b) database increased by LHS. The $\hat{\rho}$ isolines are also shown (thin lines).

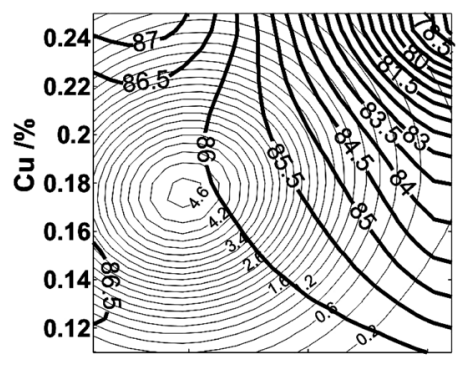

(a)

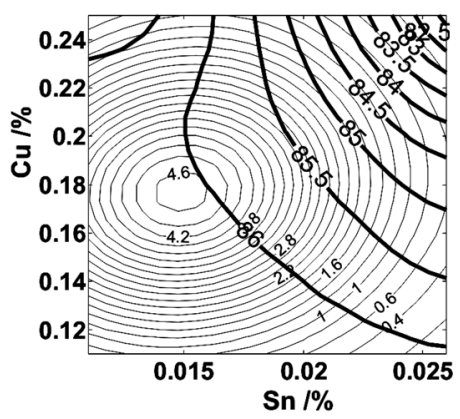

(b)

Fig. 5. Lines of equal yield $\delta$ depending on $\mathrm{Sn}$ and $\mathrm{Cu}$ contents: (a) original database, (b) database increased by LHS. The $\hat{\rho}$ isolines are also shown (thin lines). 


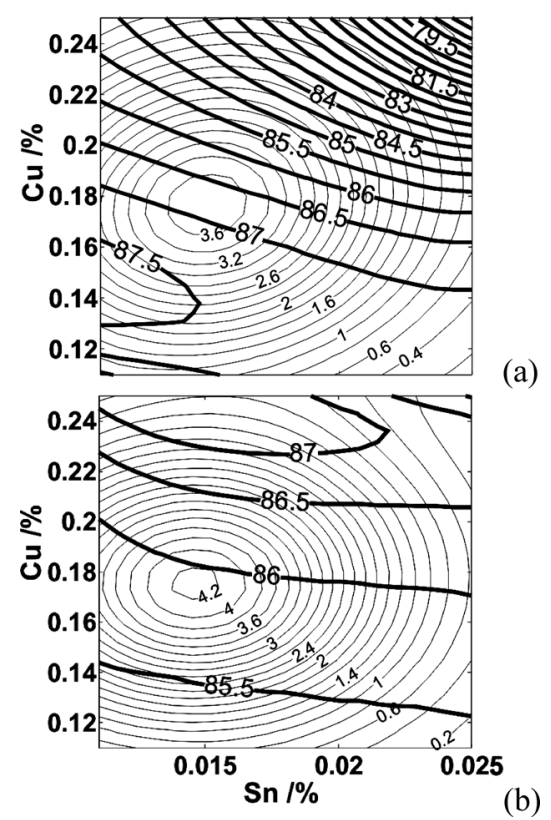

Fig. 6. Lines of equal yield $\delta$ depending on $\mathrm{Sn}$ and $\mathrm{Cu}$ at various contents of Ni: (a) $0.1 \% \mathrm{Ni}$, (b) $0.3 \% \mathrm{Ni}$, database increased by LHS. The $\hat{\rho}$ isolines are also shown (thin lines).
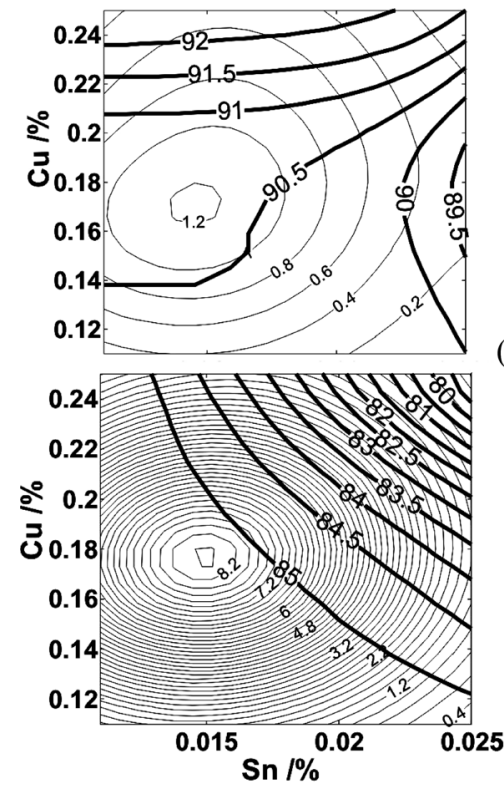

(a)

(b)

Fig. 7. Lines of equal yield $\delta$ depending on $\mathrm{Sn}$ and $\mathrm{Cu}$ at varius contents of C: (a) $1.48 \% \mathrm{C}$, (b) $1.55 \% \mathrm{C}$, database increased by LHS. The $\hat{\rho}$ isolines are also shown (thin lines).

This result regarding $\mathrm{Cr}$ is surprising since this element is carbide-forming. The thermodynamic calculations carried out by THERMOCALC showed that an increased content of $\mathrm{Cr}$ also increases the temperature of precipitation of eutectic carbides which are between $1216-1237^{\circ} \mathrm{C}$. This result is valid for thermodynamic equilibrium, but in our case the interval of precipitation is shifted to lower values, below $1200^{\circ} \mathrm{C}$, due to non-equilibrium conditions prevailing during cooling of cast ingots. Since in industrial practice the soaking temperature of ingots is approximately $1200^{\circ} \mathrm{C}$ and the first hot rolling deformations are carried out a little below this temperature, the eutectic carbides on dendritic
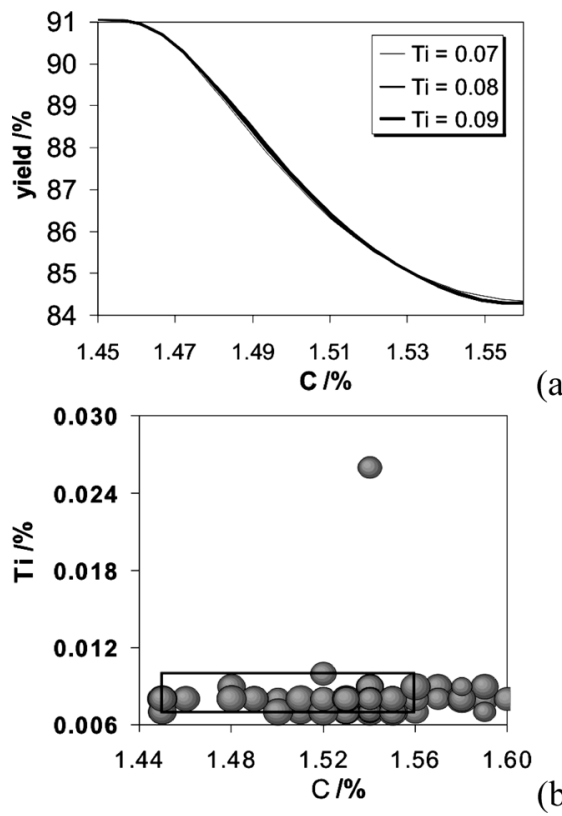

(a)

Fig. 8. (a) yield $\delta$ in relation to Ti and $\mathrm{C}$ contents, and (b) the corresponding frequency distribution of $\mathrm{Ti}$.
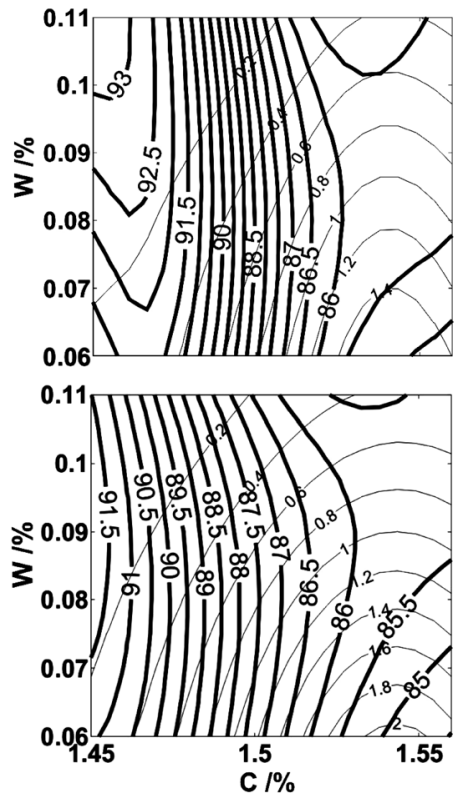

(b)

Fig. 9. Lines of equal yield $\delta$ depending on $\mathrm{C}$ and $\mathrm{W}$ contents: (a) original database, (b) database increased by LHS. The $\hat{\rho}$ isolines are also shown (thin lines).

boundaries are a function of the amount of $\mathrm{Cr}$ in either solid or in liquid state. In the last case grain boundary cracking appears due to nonadherence on dendritic boundaries. While the results of the analysis shown on Fig. 2(a) are clear in the middle part of the allowable range of variation of chemical composition due to the higher density of measured data in this interval, they appear to be less clear near the boundaries of the intervals. On the other hand, by applying the LHS method as described above the nonconsistency of the results on boundaries of the intervals are removed. This is shown on Fig. 2(b). Note that at a Cr content $(>12 \%)$ the relationship obtained by the LHS method 
gives more reasonable results. Namely, a decrease of yield $\delta$ with increase of $\mathrm{Cr}$ content, which is attributed to the increased content of carbides with increase of $\mathrm{Cr}$ content above $12 \%$.

In contrast to above obtained relationship between yield $\delta$ and $\mathrm{Cr}-\mathrm{C}$ pairs, the opposite influence of Mo in comparison to $\mathrm{Cr}$ was observed for Mo-C pairs; increasing the content of both chemical elements the yield $\delta$ decreases, which is related to Mo as carbide-forming element (see Fig. 3(a)).

$\mathrm{CoV}$ for $\mathrm{Mo}$ and $\mathrm{C}$ amount to $1.3 \%$ and $0.7 \%$, respectively, thus with regards to the results obtained for the industrial and LHS extended database also in this case a small difference in results obtained from the industrial database in comparison to the LHS extended database was found (see Fig. 3(b)). Thus lower values of $\mathrm{CoV}$ for $\mathrm{Cr}$, Mo and $\mathrm{C}$ in general result in a lower difference between results obtained by analysis of only measured data and LHS increased data, respectively.

The results of the analysis of the influence on yield $\delta$ shown in Figs. 4-7 are for chemical elements for which $\mathrm{CoV}$ lies in the moderate range. Similar relationships were also obtained for Mn-S pairs. From Fig. 4(a) it is clear that an increase in sulphur content at the same level of Mn decreases the yield $\delta$ of hot rolling due to increased surface cracking. On the contrary, an increase in the content of Mn at the same level of sulphur increases the yield $\delta$ of hot rolling. It is also apparent that at the upper and lower limits of the range of variation of sulphur the relationships are not very clear. A higher degree of discontinuity of the relations in comparison to that obtained in the middle of the variation range is evident. The discontinuity can be ascribed to the uncertainty, and to a smaller extent, also to the data distribution at the boundaries of the range of variation. This was again improved using the LHS method, as can be seen from Fig. 4(b).

The presence of $\mathrm{Cu}$ and $\mathrm{Sn}$ in increased contents that deteriorate hot deformability is very frequent when tool steels are produced from scrap. Thus the analysis of their negative influence and the possibility of decreasing their negative effects should be carried out. An analysis of their influence on yield $\delta$ is shown on Fig. 5. An increase in $\mathrm{Cu}$ content at the lowest values of $\mathrm{Sn}$ does not result in a decrease of the yield $\delta$, while with increase of Sn content, despite the lower content of $\mathrm{Cu}$, the yield $\delta$ also decreases. Thus we can conclude that the presence of $\mathrm{Sn}$ at the lowest contents is not harmful for the yield $\delta$, even though $\mathrm{Cu}$ is simultaneously present in higher concentrations. Namely, $\mathrm{Sn}$ and $\mathrm{Cu}$ form a phase with a low melting point that accelerates grain boundary cracking since it precipitates on the grain boundaries. ${ }^{5)}$ Figure 5(b) again demonstrates that using LHS improves the relationships at the upper and lower limits of allowable variation of $\mathrm{Sn}$.

According to the results for carbon steels obtained by Imai et al., ${ }^{6}$ Matsuoka et al., ${ }^{7)}$ and also in the present study for tool steel, Ni could decrease the negative influence of $\mathrm{Cu}$ and $\mathrm{Sn}$ when they are present in steel simultaneously. In Fig. 6(a) the influence of $\mathrm{Cu}$ and $\mathrm{Sn}$ on yield $\delta$ at $0.1 \%$ of $\mathrm{Ni}$ is presented. With increased content of $\mathrm{Sn}$ at the same level of $\mathrm{Cu}$ content a smaller decrease of yield $\delta$ can be observed than for the case where the average value of Ni content is considered (implicitly) (see Fig. 5). Furthermore,
Imai et $\left.a{ }^{6}{ }^{6}\right)$ found that addition of $0.3 \%$ of $\mathrm{Ni}$ to steels, where $\mathrm{Cu}$ and $\mathrm{Sn}$ are present simultaneously, increases the hot ductility of studied steels. The same was observed in the present study, as the influence of Sn decreased when the Ni content was $0.3 \%$. This can be clearly seen on Fig. 6(b) where the inclination angles of the isolines with equal yield $\delta$ are small. Furthermore, in comparison with Fig. 6(a) where the yield $\delta$ decreases with increasing $\mathrm{Cu}$ content, the opposite behaviour was observed in Fig. 6(b). It was also found by a trial end error procedure that the transition from the state presented in Fig. 6(a) to the state presented in Fig. 6 (b) occurs at a Ni content of $0.2 \%$.

The influence of $\mathrm{C}$ content when $\mathrm{Cu}$ and $\mathrm{Sn}$ are present simultaneously in steel was studied by Matsuoka et al. ${ }^{7)}$ in their laboratory examination of hot deformability by hot tensile tests. They observed a decrease in hot ductility with increasing $\mathrm{C}$ content. The influence of $\mathrm{C}$ was also studied in the present investigation and similar trends were observed, as shown on Fig. 7 for $1.45 \%$ and $1.55 \%$ contents of C. For a lower content of $\mathrm{C}$ (Fig. 7(a)) the influence of $\mathrm{Sn}$ and $\mathrm{Cu}$ on yield $\delta$ is less significant than for a higher amount of $\mathrm{C}$ (Fig. 7(b)). Also in this case a transition between the two regimes was observed for a $\mathrm{C}$ content of approx. $1.5 \%$. Namely, for higher $\mathrm{C}$ content the yield $\delta$ decreases with increasing content of $\mathrm{Sn}$ and $\mathrm{Cu}$, and for a higher amount of $\mathrm{C}$ the opposite is true.

In the steels examined there were also very small amounts of $\mathrm{W}$ (about $0.01-0.06 \%$ ) present, as well as $\mathrm{Ti}$ (about $0.07 \%$, the $\mathrm{CoV}$ for $\mathrm{Ti}$ was thus high about $123 \%$ ). $\mathrm{Ti}$ is a strong carbide-forming element that forms mainly MC-type of carbides. Thus the variation range of Ti was too narrow to allow the presentation of its influence on hot deformability in the same way. Figure 8(a) makes it clear that the yield $\delta$ increased by $5 \%$ if the carbon content was reduced from 1.56 to $1.45 \%$, even though the Ti content was very low. On Fig. 8(b) the distribution frequency of the data is shown. These results are in agreement with the results obtained by Sandberg, 1973, who found that Ti, though present in very small concentrations (about 0.01\%), increased the degree of micro-segregation.

The CoV for $\mathrm{W}$ was also high (about 79\%). A comparison between the results obtained with the original and LHS enhanced database for $\mathrm{W}-\mathrm{C}$ pairs and yield $\delta$ are shown on Fig. 9. It can be seen that increasing the carbon content decreases the yield $\delta$. It is also evident that $\mathrm{W}$ in the range of approx. $0.06-0.11 \%$ has no essential influence on yield $\delta$, but in the lower range of variation this continuity is lost. Using LHS the relationships at the upper and lower limits of allowable variation of $\mathrm{W}$ are clearer and the continuity, regarding the middle part of the variation range, was improved (see Fig. 9(b)).

Greater improvements are obtained for chemical elements with a higher $\mathrm{CoV}$. The results obtained demonstrate that the absolute predicted values within the area of higher reliability are very similar for both approaches (original database vs. LHS extended database). In the latter case the results are smoother and physically clearer. Note that smoother results may also be obtained by increasing $w$, but in this case quantity is lost due to the better quality (physical clearness). By introducing the LHS procedure, the problem is solved-one can obtain both in one solution: reason- 
ably quantitative results and physical clearness. The results obtained were also directly transferred into industrial practice, resulting in a $c a$. $15 \%$ increase of yield $\delta$.

\section{Conclusions}

The Latin hypercube sampling (LHS) technique was successfully applied in order to overcome the problem of a relatively small data base and the uncertainty of measurements in the process of hot deformation of tool steels. The advantage of this approach was demonstrated in the analysis of the influence of chemical composition on yield $\delta$ during hot rolling of AISI D2 tool steel. By using the LHS technique the accuracy of analysis was improved, especially close to the lower and to upper limits of the allowable variation range. The relationships obtained between influential parameters and hot deformability enable the yield $\delta$ in industrial production process to be increased by up to $15 \%$. In general, using LHS, the changes in relationship obtained were greater for chemical elements with a higher $\mathrm{CoV}$. Ti, despite its small content, considerably influences hot deformability. An increased S content results in a decreased yield $\delta$, but on the contrary, an increased Mn content results in an increased yield $\delta$. A strong influence of Sn in relation to $\mathrm{Cu}$ on the hot deformability at values higher than $0.02 \%$ was observed. The negative influence of $\mathrm{Cu}$ and $\mathrm{Sn}$ on yield $\delta$ can be eliminated with an appropriate content of $\mathrm{Ni}$. Increasing the $\mathrm{Cr}$ content up to approximately $12 \%$ increases the yield $\delta$ since it increases the temperature of precipitation of eutectic carbides. On the other hand, increasing the content of Mo decreases the yield $\delta$.

\section{REFERENCES}

1) J. Majta, R. Kuziak and M. Pietrzyk: J. Mater. Process. Technol., 80-81 (1998), 524.

2) T. Večko Pirtovšek, G. Kugler, P. Fajfar, M. Fazarinc, I. Perus and M. Tercelj: RMZ-Materials and Geoenvironment, 54 (2007), No. 1, 1.

3) T. Večko Pirtovšek, G. Kugler, M. Fazarinc and M. Terčelj: RMZMaterials and Geoenvironment, 54 (2008), No. 2, 147.

4) C. Rodenburg, M. Krzyzanowski, J. H. Beynon and W. M. Rainforth: Mater. Sci. Eng. A, 386 (2004), No. 1-2, 420.

5) N. Imai, N. Komatsubara and K. Kunishihe: ISIJ Int., 37 (1997), No. 3,224 .

6) N. Imai, N. Komatsubara and K. Kunishihe: ISIJ Int., 37 (1997), No. $3,217$.

7) H. Matsuoka, K. Osawa, M. Ono and M. Ohmura: ISIJ Int., 37 (1997), No. 3, 255.

8) C. A. C. Imbert and H. J. McQueen: Can. Metall. Q., 40 (2001), 235.

9) J. Sinzcak, J. Majta, M. Glowacki and M. Pietrzyk: J. Mater. Process. Technol., 80-81 (1998), 166.

10) M. Pietrzyk: J. Mater. Process. Technol., 125-126 (2002), 53.

11) L. A. Dobrzanski, M. Kowalski and J. Majdejski: J. Mater. Process. Technol., 164-165 (2005) 1500.

12) M. Çöl, H. M. Ertunç and M. Yılmaz: Mater. Des., 28 (2007), No. 2, 488.

13) C. Capdevila, C. Garcia-Mateo, F. G. Caballero and C. Garcia de Andres: Comput. Mater. Sci., 38 (2006), No. 1, 192.

14) H. Sandberg: Scand. J. Metall., 2 (1973), 233.

15) W. Sha and K. L. Edwards: Mater. Des., 28 (2007), No. 6, 1747.

16) Z. Guo and W. Sha: Comput. Mater. Sci., 29 (2004), No. 1, 12.

17) M. Terčelj, R. Turk, G. Kugler, I. Perus: Comput. Mater. Sci., 42 (2008), 625.

18) S. Mandal, P. V. Sivaprasad, S. Venugopal, K. P. N. Murthy and B. Raj: Mater. Sci. Eng. A, 485 (2008), No. 1-2, 571.

19) I. Grabec and W. Sachse: Synergetics of Measurement, Prediction and Control, Springer-Verlag, Berlin Heidelberg, (1997), 205.

20) M. D. McKay, W. J. Conover and R. J. Beckman: Technometrics, 21 (1979), 239.

21) R. L. Iman, J. C. Helton and J. E. Campbell: J. Quality Technol., 13 (1981), No. 3, 174

22) I. Peruš, P. Fajfar and K. Poljanšek: Earthquake Eng. Struct. Dyn., 35 (2007), No. 12, 1453

23) R. Turk, I. Perus and M. Terčelj: Int. J. Machine Tools Manufacture, 44 (2004), No. 12-13, 1319. 